\title{
A novel remote access technical database for the offshore diving industry
}

\author{
D. Johnston ${ }^{1}$, T. Henaughen ${ }^{1}$, B. G. Stewart ${ }^{2}$, A. Nesbitt ${ }^{2}$ \\ \& A. Ahmadinia ${ }^{2}$ \\ ${ }^{1}$ Shearwater Marine Services Ltd., Scotland, UK \\ ${ }^{2}$ Glasgow Caledonian University, Scotland, UK
}

\begin{abstract}
The commercial diving industry has largely resisted efforts to implement digital information management systems. The high cost and inherent difficulty of creating not only the necessary software systems, but hardware which is capable of operating in harsh marine conditions has proved to be a major obstacle. Further to this is the challenge of creating data connections between centralised data stores and offshore field units, or units in remote areas with limited access to internet infrastructure. Dive logs are commonly taken by hand, requiring several stages of transcription and transport over days or weeks before they can be communicated to clients. With minimal standards in place regarding standardised terminology the consistency of reports can vary not only between marine contractors, but between individuals within these organisations. The lifesupporting nature of many marine structures renders this type of miscommunication potentially expensive and potentially life-threatening.

This paper outlines one approach to these problems through amalgamating existing communications, database and software technologies to provide a system which addresses the needs of the industry. This approach has provided the capability to manage dive teams both within the UK and globally. Real-time video streaming of subsea operations combined with software data capture tools, rugged hardware and integrated database technologies allows remote teams to communicate with clients and industry specialists while on-site. Clients can also secure remote access to a technical database post-dive where they are able to view video streams and associated key technical information related to the dive. The benefits and added value of the system to the stakeholders are examined, along with the applicability of the system to broader industry. Keywords: database systems, video technology, remote access.
\end{abstract}




\section{Introduction}

The marine environment is inherently inhospitable to digital communications technology. Remote locations with little or no data connectivity, variable temperatures, humidity and salt $[1,2]$ combine to create a 'perfect storm' of undesirable conditions for IT projects. As a result of this the commercial diving industry has lagged behind other sectors in terms of its adoption of digital infrastructure, and the various benefits that come with it. With an ever-growing demand for high resolution streaming video on off-shore platforms [3], this is set to change in the imminent future.

Information gathered during dives is commonly recorded using pen and paper, in an analogous fashion to that observed in fisheries by Barkail [4]. While this approach is certainly low-cost and minimally intrusive to on-going operations, it significantly increases the difficulty of effectively managing both this data and the key information derived from the data. At a minimum, handwritten notes must be accurately transcribed to a digital format, transmitted to a central office, proofread and re-formatted before being issued to the end client. Similarly, video footage created during dives is often recorded to DVD or, more recently, hard disk or SD cards. These are physically transported to a central office before being duplicated for distribution to clients. This process is extremely time-consuming, prone to human error [5], and complex to manage efficiently. Even after its completion the results can be unsatisfactory, offering limited opportunities for data mining and no cohesive mechanism to exploit the potentially valuable information gathered.

Beyond the practical implications of operating almost entirely offline are the strong business drivers for implementing a more sophisticated technology based platform for data management. A well-managed central data store can provide numerous benefits such as improved knowledge of client requirements, better decision making, improved operational efficiency and reduced costs among others [6]. Given the lack of this type of technology in the current commercial diving industry there are significant opportunities to create entirely new revenue streams from services such as real-time video streaming from on-going dives and online resources for clients which allow 'always on' access to digital assets collected on their behalf.

With recent advances in rugged PC hardware and wireless data infrastructure many of the data management issues facing the diving industry can now be addressed. This paper examines one development and implementation approach to these issues for a small-to-medium enterprise (SME) within the diving industry, outlining a system which incorporates rugged computing hardware, satellite data transmission and proprietary software assets to provide an integrated data management platform.

\section{Background}

Shearwater Marine Services, a commercial diving contractor based on the west coast of Scotland, commissioned the development of a system which would 
provide them with an end-to-end data management solution. The requirements for this system incorporated elements which would address the issues outlined above, facilitating rapid data transportation, terminology standardisation, remote working and long term digital storage.

\section{Implementation}

\subsection{Architecture}

The system developed consists of four separate software applications, described in Table 1, and shown within a general operational system architecture in Figure 1.

Table 1: $\quad$ Developed software applications.

\begin{tabular}{|l|l|}
\hline Data server application & $\begin{array}{l}\text { Server-side application which controls access to the central } \\
\text { relational database and provides a web interface for clients }\end{array}$ \\
\hline Video server application & $\begin{array}{l}\text { Server-side application which provides access to video footage } \\
\text { recorded during dives }\end{array}$ \\
\hline Dive recorder application & $\begin{array}{l}\text { Native desktop application which provides video } \\
\text { recording/streaming and data logging facilities }\end{array}$ \\
\hline Office based application & $\begin{array}{l}\text { Native desktop application which provides task management } \\
\text { facilities }\end{array}$ \\
\hline
\end{tabular}

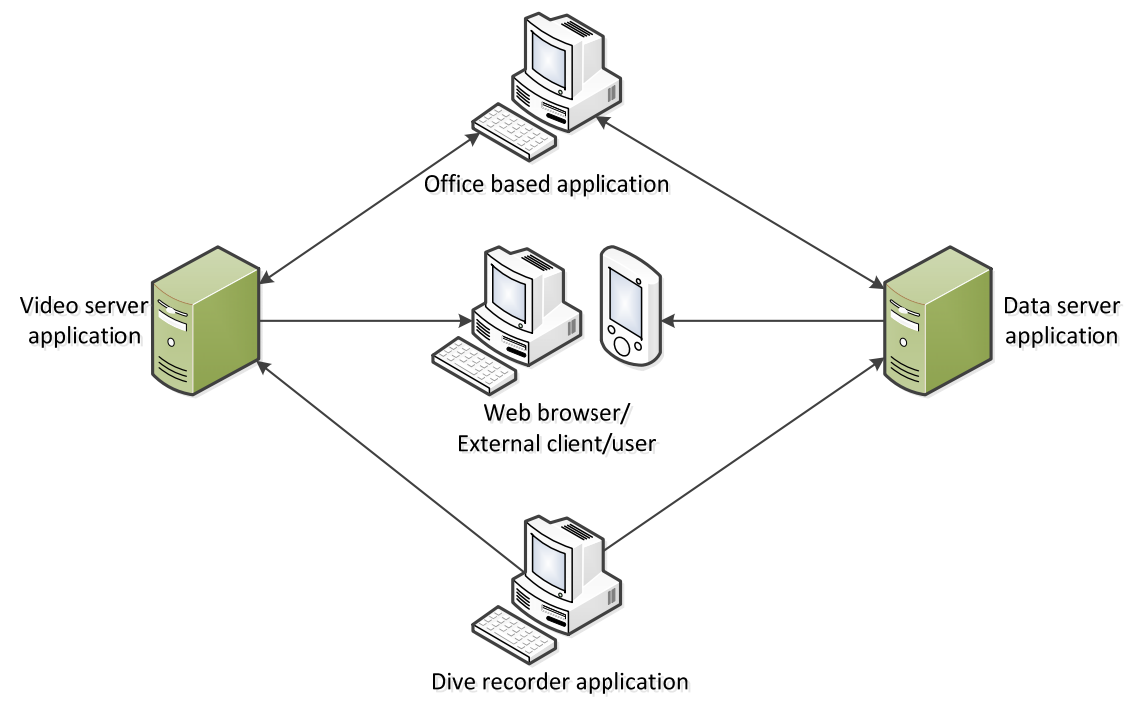

Figure 1: $\quad$ System architecture.

The applications listed in Table 1 are described as follows. 


\subsection{Data server application}

The data server application is effectively the core of the system hosting the fundamental database developments. Developed using Microsoft's Model-ViewController (MVC) Framework in conjunction with the Entity Framework, it contains a set of classes which define the relational database schema and are used to create the database on the application's first run. Access to the SQL Server relational database is provided to both the dive recorder and office based applications using Windows Communication Foundation (WCF) Data Services which expose the software objects defined in the database generation classes over the OpenData (OData) protocol.

In addition to managing access to the relational database the data server application also provides a basic secure web interface. This can be used by clients and company staff to monitor task schedules, and access all information captured during previous dives. Figure 2 shows a view of the video review user interface screen which allows the client to examine recorded video footage, along with the timestamped logs captured during the task. Each of the logs displayed are clickable and cause the video to seek to the point in time indicated next to the log. This is a significant improvement over previous methods of video review, which involved manually seeking through DVD recordings and was extremely time consuming.

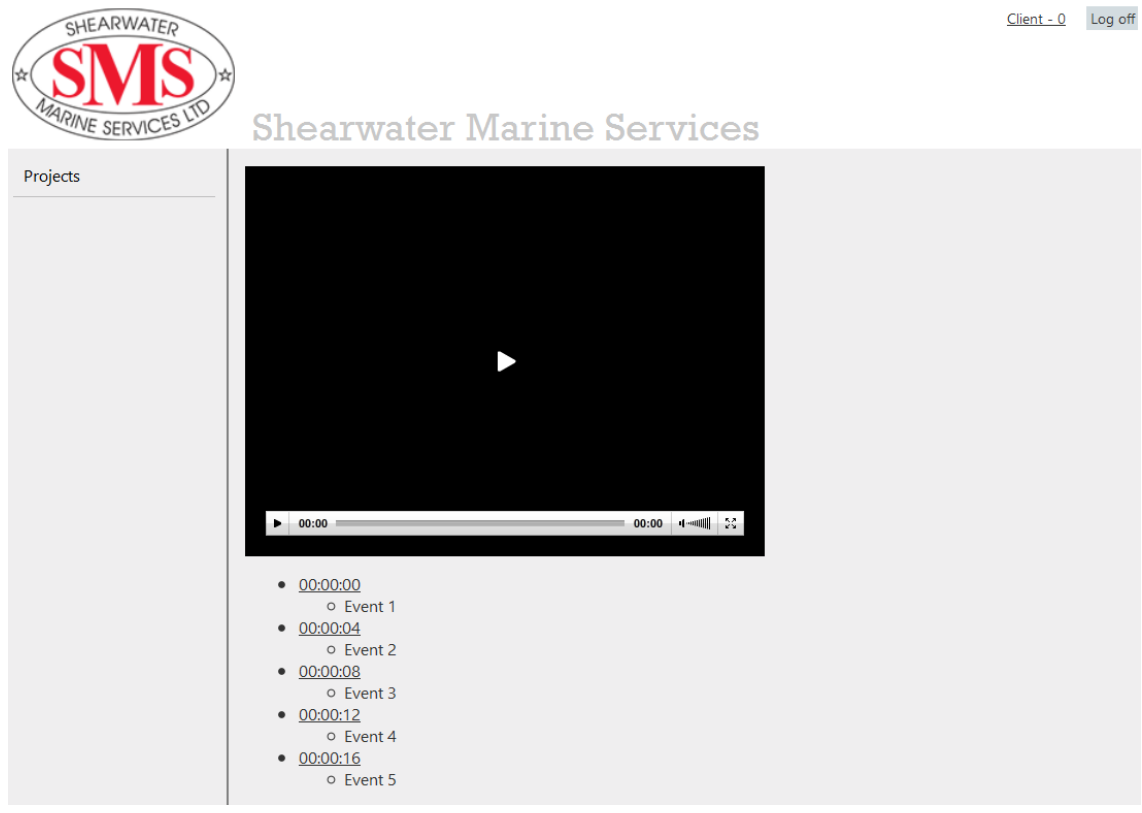

Figure 2: Web application interface.

The final facility provided by the data server application allows the user to generate and download a report, formatted as a Microsoft Word document, 
containing all of the information captured during a given task. This automation eliminates the need for the diving contractor to physically locate, duplicate and re-issue the original report.

\subsection{Video server application}

This application provides facilities for securely sending and receiving streaming audio and video to multiple simultaneous clients over the internet. Wowza Server 3.5 was chosen as the video server application for this project as it offers a wide range of media streaming capabilities at relatively low cost. Wowza was configured to receive and re-broadcast incoming live video streams from the dive recorder application, allowing users to view on-going dive operations in realtime via the system's web interface. In addition to its live streaming capability Wowza provides Video-On-Demand (VOD) access to the high resolution footage uploaded by dive teams at the end of a task. Any footage uploaded is stored on the same remote server as the instance of Wowza and can be retained indefinitely for archival purposes. Over a period of several years this should allow the diving contractor to create an invaluable long term asset monitoring resource.

\subsection{Office based application}

This allows users to effectively manage task creation and review data collected from dives. Developed using the .Net Windows Presentation Foundation (WPF) Framework, this application consumes the OData feed provided by the data server application.

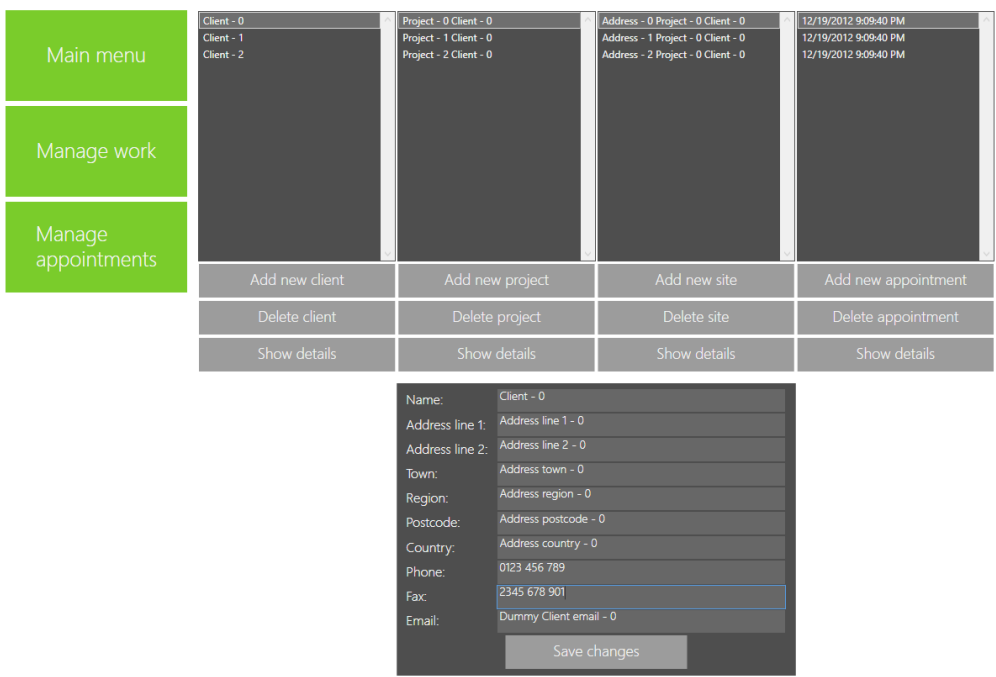

Figure 3: $\quad$ Office based application. 
The interface shown in Figure 3 allows the user to manage the information relating to on-going tasks, such as dive site locations and task schedules. Adding a new task using this interface creates a placeholder entry in the relational database in readiness for data to be uploaded by the dive team assigned to the task. On completion of a task which has been set up using the interface shown in Figure 3 the assigned dive team uploads all recorded logs and video. These can then be reviewed and edited by staff working at the contractor's central office using a further user interface shown in Figure 4.
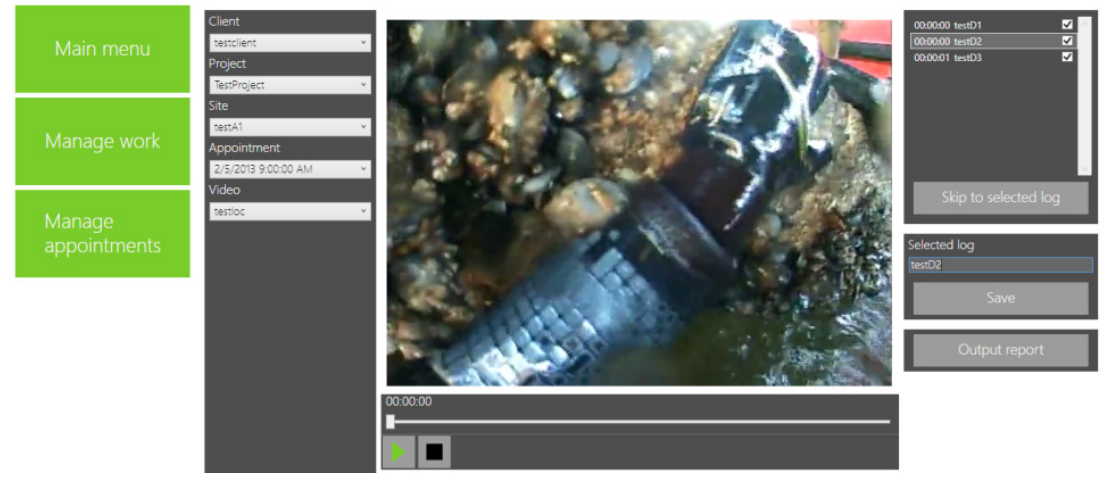

Figure 4: Video review interface.

After the dive data has been edited and approved by a member of staff from the diving contractor it is published to the web interface provided by the data server application.

\subsection{Dive recorder application}

The dive recorder application is intended for use by dive supervisors during active dive tasks. As with the office based application, this has been developed using the WPF Framework in order to leverage the full hardware capabilities of its host machine. It allows the user to simultaneously record high resolution H.264 format video to a local hard disk and, where internet connectivity is available, stream it to the video server application using the Real Time Protocol (RTP). The H.264 codec provides the means to capture these high resolution recordings with relatively low bandwidth and disk space consumption. In addition to this H.264 features a wide range of configurable options which can be employed to optimise the video recording to a high degree while maintaining a low bandwidth profile.

The main interface of the recording software is shown in Figure 5. On discovery of a noteworthy item during the dive task the dive supervisor creates a new log which is timestamped with the elapsed video recording time. This timestamp provides the mechanism for users to rapidly seek through the video footage when displayed in the office and web based applications. All log data is stored locally in an XML file prior to being uploaded to the remote data server on the completion of the dive task. 


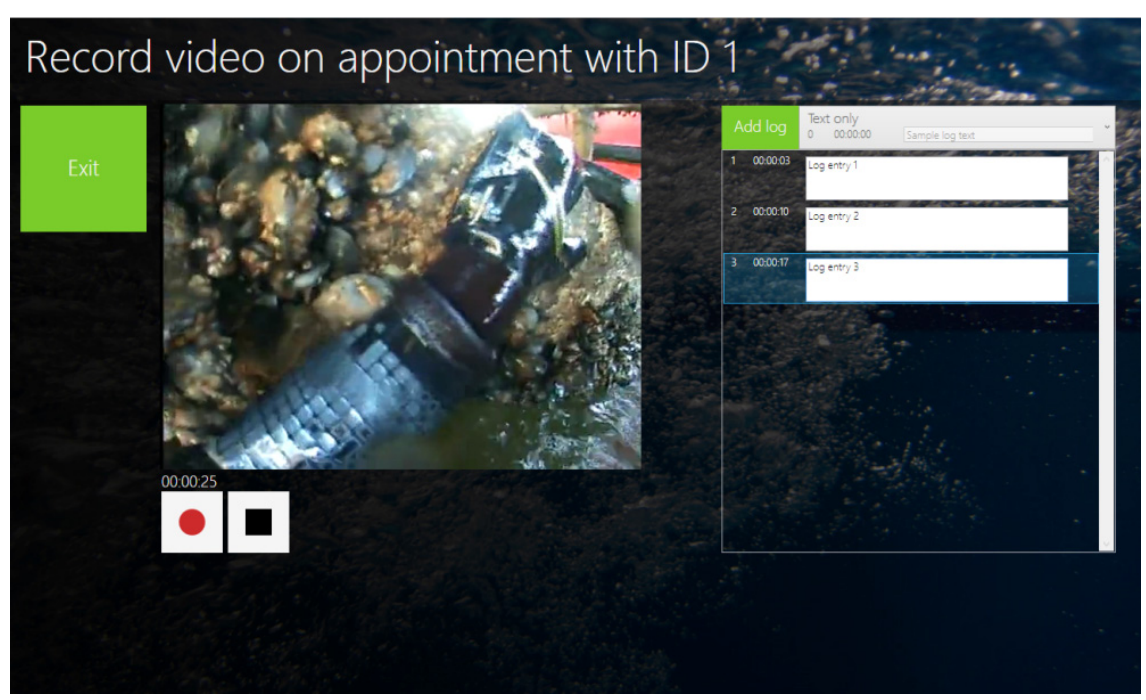

Figure 5: $\quad$ Main dive recorder interface.

On completion of a dive task the user is required to upload all captured logs and video footage to the remote data and video servers respectively. On the successful completion of this upload the materials become available for review by managerial staff before publication for the consumption of client users.

\section{Conclusions and future work}

The developed system is currently in active beta trials with a limited user group prior to a full commercial roll-out. Early indications from the client user group suggest that the proposed system is of significant benefit. The main advantages of the system may be summarised as follows:

\subsection{Cost reduction}

The transition from physical to digital media will provide significant cost reductions in terms of media storage and man-hours spent on duplicating, transcribing and transporting physical media assets. Additional savings will be realised by providing clients with the means to access their own data freely, and without the intervention of staff members.

\subsection{Reduced turnaround time}

With video being transmitted in real-time during diving operations, and log data being uploaded to a central server immediately following tasks, the time taken for critical data to reach stakeholders is significantly reduced. This data can now be made available to all parties within hours of a dive completion, as opposed to the previous situation which involved delays of up to several weeks. 


\subsection{Improved client access}

The real-time streaming video facility of the system can allow client representatives to view dives from their own facilities, as opposed to dispatching a supervisor to the site. This is extremely attractive to many organisations, as dispatching staff to remote locations is both time consuming and costly. Further to this, technical experts located anywhere in the world can now view on-going tasks and provide valuable oversight and comment.

\subsection{Data mining}

As data accumulates in the system, it becomes an ever more valuable resource to both clients and contractor. Long term condition assessments can now be carried out quickly and at low cost by analysing data from a given structure over a period of several years. Where previously this would have involved manually collating data from physical records, the creation of a central database allows its operators to quickly identify, retrieve and organise information relevant to any previous task.

The software applications developed to date show much promise, and will be further extended to incorporate more business management tools. Integrating the existing task scheduling tools with a Planned Maintenance System (PMS) will allow the contractor to monitor equipment and human resource allocation. This integration will also allow the collection of data on resource efficiency, and improve the ability of the contractor to manage on-going diving operations.

\section{Acknowledgement}

This work was supported by a UK Knowledge Transfer Partnership (KTP) Grant (KTP007945) awarded to Shearwater Marine Services and Glasgow Caledonian University.

\section{References}

[1] Natesan, M., Corrosion behavior of metals and alloys in marine-industrial environment. Science and technology of advanced materials, 9(4), 2008

[2] Rajan Ambat, Per Møller, Corrosion investigation of material combinations in a mobile phone dome-key pad system, Corrosion Science, Volume 49, Pages 2866-2879, 2007

[3] http://www.satellitetoday.com/via/cover/Video-in-an-Offshore-Environment -Fuelling-Bandwidth-Demands_39735.html

[4] Barkail, A., The development and implementation of eLog technology for the local and international fishing industry, IST-Africa Conference Proceedings, 2011 , pp. 1-15

[5] Logan, F.A., Errors in Copy Typewriting, Journal of Experimental Psychology: Human Perception and Performance [PsycARTICLES], vol. 25, no. 6, pp. 1760-1760, 1999

[6] Loshin, D., Master Data Management, Morgan Kaufmann Publishers Inc., San Francisco, CA, 2008 